\title{
Photogenerating Silver Nanoparticles and Polymer Nanocomposites by Direct Activation in the Near Infrared
}

\author{
Lavinia Balan,, ${ }^{1}$ Raphael Schneider, ${ }^{2}$ Colette Turck, ${ }^{1}$ Daniel Lougnot, ${ }^{1}$ \\ and Fabrice Morlet-Savary ${ }^{3}$ \\ ${ }^{1}$ Institut de Science des Matériaux de Mulhouse, CNRS LRC 7228, 15 rue Jean Starcky, 68057 Mulhouse, France \\ ${ }^{2}$ Laboratoire Réactions et Génie des Procédés, CNRS UPR 3349, Nancy-University, 1 rue Grandville, 54001 Nancy, France \\ ${ }^{3}$ Department of Photochemistry, CNRS FRE 3252, Haute Alsace University, 3 rue A. Werner, 68100 Mulhouse, France
}

Correspondence should be addressed to Lavinia Balan, lavinia.balan@uha.fr

Received 8 August 2011; Accepted 6 September 2011

Academic Editor: Sevan P. Davtyan

Copyright (C) 2012 Lavinia Balan et al. This is an open access article distributed under the Creative Commons Attribution License, which permits unrestricted use, distribution, and reproduction in any medium, provided the original work is properly cited.

\begin{abstract}
This work reports on an improvement of the photochemically assisted synthesis of silver nanoparticles by direct photoreduction of $\mathrm{AgNO}_{3}$ with a laser source emitting in the near infrared range (NIR). For this, polymethine dyes were used as the photoactive agents. Both the effects of central chain structure and activation intensity were investigated. The reduction kinetics was followed up by UV-Vis spectroscopy, and the particles size was evaluated by transmission electron microscopy. The results showed that light intensity affects both the average size and size distribution of Ag nanoparticles generated through this process. The particles can also be generated in situ in a photopolymerizable formulation so that metal/polymer nanocomposites become available through a one-step photoassisted process on the basis of NIR activation. The process described herein is very fast (seconds to a few minutes), and it readily lends itself to automatization for mass production of micro-optical elements implemented directly onto integrated NIR sources.
\end{abstract}

\section{Introduction}

Recent developments in optics and photonics require novel, simple, and fast methods to fabricate metal nanoparticles (MNPs). In recent years, a whole bunch of synthetic methods for the preparation of MNPs have been developed: chemical, photochemical, and thermal. Amongst them, the photochemical synthesis of MNPs including direct photoreduction and photosensitization has attracted intense research interest, since it is a versatile and convenient process with distinguishing advantages such as space selectivity [1].

Embedding nanosized MNPs into polymer matrixes is also of great interest, because these materials combine properties from both inorganic and organic systems. Thus, MNPs homogeneously dispersed in polymer matrixes are already used as sensors [2-4], materials with solvent switchable electronic properties [5], optical limiters or filters [6, $7]$, optical data storage materials $[8,9]$, surface Plasmonenhanced random lasing media [10], catalytic additives [11], or antimicrobial coatings $[12,13]$.
Metal-polymer nanocomposites are usually obtained via multistep methods. Dry silver NPs produced beforehand can be dispersed into a polymerizable formulation to obtain self-assembly functionalized structures. However, besides the specific hazards related to handling dry NPs, their size dispersity over a large scale is difficult to control, thus limiting the interest of this "ex situ" method $[14,15]$.

The "in situ" approach that involves the generation of MNPs directly in a polymerizable medium through reduction of a cationic precursors offers the advantages of better dispersion ability and facile chemical or photochemical reduction $[16,17]$.

Several examples of in situ synthetic routes to MNPs and polymer/metal nanocomposite were reported as yet and the formulations used contain a variety of monomers and a collection of photoinitiators/photosensitizers [18-22]. They highlighted the flexibility in terms of temperature, dispersion, and rapidity of the process used to trigger the (photo)chemically assisted reduction of metal precursors. 
S1<smiles>CCN1/C(=C\C=C2/CC/C(=C\c3sc4ccc(Cl)cc4[n+]3CC)C2=Nc2ccccc2)Sc2ccc(Cl)cc21</smiles>

S2

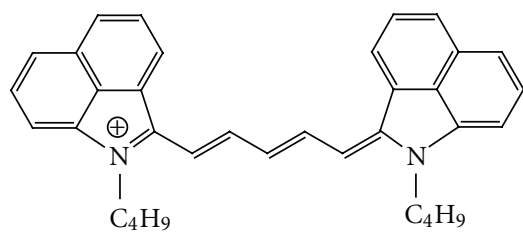

$\mathrm{BF}_{4}^{\ominus}$

FIGURE 1: Structures of the sensitizers S1: (5,5' -dichloro-11-diphenylamino-3,3' -diethyl-10,12-ethylenethiatricarbocyanine perchlorate) and S2: (1-Butyl-2-[5-(1-butyl-1H-benzo[cd]indol-2-ylidene)-penta-1,3-dienyl]-benzo[cd]indolium tetrafluoroborate) and of the coinitiator (N-methyldiethanolamine).

In our recent studies, we have reported a novel approach for the preparation of metal-polymer nanocomposites in which MNPs were obtained by direct UV or visible photoreduction [20-22]. This powerful in situ approach, involving irradiation of an appropriated formulation, induces a homogeneous distribution of MNPs in a crosslinked polymer network.

However, some recent developments in the field of applied micro-optics or nanofabrication turn round to producing highly integrated devices, and whenever the corresponding fabrication processes include photochemical steps, smallscale and low-cost light sources with low power consumption are preferred.

In another respect, scientists involved in fundamental plasmonics are demanding systems capable of generating MNPs in situ through photochemical processes that could be triggered by NIR light sources. And lastly, the possibility of generating MNPs - with bactericidal or antimicrobial activity-directly in a living medium or as a thin film polymer top coating does not fail to stimulate interest in the field of microbiology. However, the spectral window in which the photogeneration can be carried out is often restricted to the far red or NIR, because the absorption of the sample itself obstructs the other regions of the spectrum.

In this context, the present paper deals with the photochemically assisted fabrication of silver nanoparticles in situ in a polymerizable medium using a near infrared (NIR) source. Thus, the challenge consisted of tailoring a currently used process so that it could be activated with laser diodes (and even VCSELs) emitting in the 750 to $900 \mathrm{~nm}$ range instead of sources emitting in the visible or near UV.

So far, this report is the first to deal with the synthesis of silver nanoparticles and polymer-metal nanocomposites through an NIR-assisted photoprocess. Even though some examples of NIR resins were reported during the past decade [23-25], pushing the sensitivity limits of the process, generating silver nanoparticles in situ up to ca $900 \mathrm{~nm}$, turns out to be an important breakthrough.

\section{Experimental}

The formulations used in this work contained a sensitizer absorbing in the NIR (5,5'-dichloro-11-diphenylamino3,3' -diethyl-10,12-ethylenethiatricarbocyanine perchlorate, (S1) and 1-Butyl-2-[5-(1-butyl-1H-benzo[cd] indol-2-ylid ene)-penta-1,3-dienyl]-benzo[cd]indolium tetrafluoroborate, (S2) from Sigma-Aldrich) associated with a coinitiator, $\mathrm{N}$-methyldiethanolamine (MDEA) from Sigma-Aldrich and a triacrylic monomer (pentaerythritoltriacrylate from Sartomer). The chemical structures of the sensitizer and cosensitizer are shown on Figure 1. The precursor of silver nanoparticles was silver nitrate (ACS Reagent 99\% from Aldrich). It was added to the formulation under stirring and maintained one hour before use.

The mechanism used to produce silver nanoparticles and initiate polymerization is the following: upon NIR irradiation, the dye sensitizer which absorbs in the 750$900 \mathrm{~nm}$ range (see Figure 2) is photoexcited and reacts with the coinitiator (1). Amine-derived radical species $\left(\mathrm{RH}^{\bullet+}\right)$ is capable of inducing reducing of silver cations (2) and freeradical polymerization of the monomer (M) (3)

$$
\begin{gathered}
\text { Sens }+h \nu \longrightarrow \text { Sens }^{*} \\
\text { Sens }^{*}+\mathrm{RH} \longrightarrow \text { Sens }^{\bullet-}+\mathrm{RH}^{\bullet+} \\
\mathrm{RH}^{\bullet+} \longrightarrow \mathrm{R}^{\bullet}+\mathrm{H}^{+} \\
\mathrm{R}^{\bullet}+\mathrm{Ag}^{+} \longrightarrow \mathrm{Ag}^{0}+\mathrm{X} \\
\mathrm{R}^{\bullet}+\mathrm{M} \longrightarrow \text { Living polymer radical }
\end{gathered}
$$




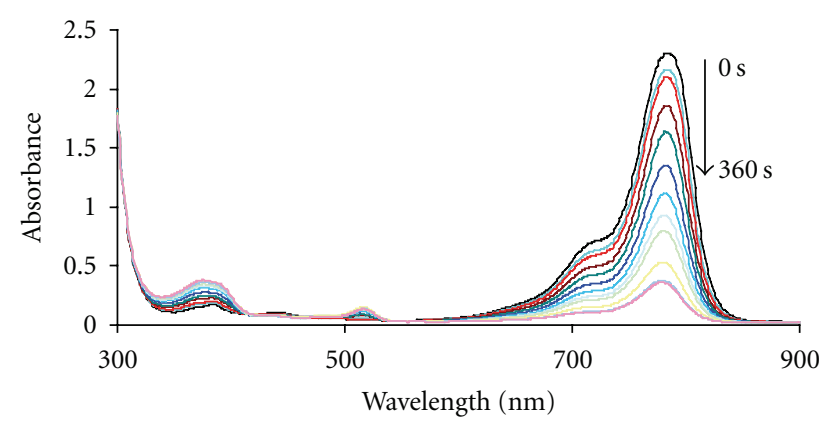

(a)

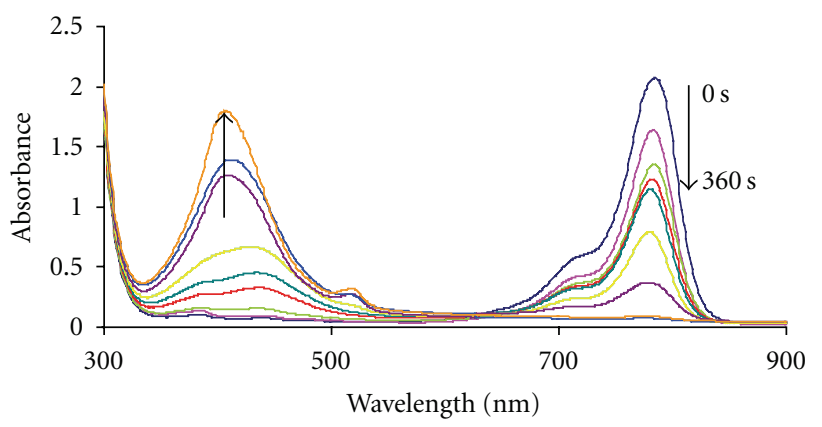

(b)

Figure 2: Time evolution of absorption spectra of the samples with S1 upon irradiation at $800 \mathrm{~nm}$ : (a) without silver cations and (b) with silver cations. Incident light power was $300 \mathrm{~mW} / \mathrm{cm}^{2}$ in laminated conditions, and irradiation times were chosen between 0 and $360 \mathrm{sec}$.

This process is known to be very sensitive to oxygen quenching [26]. For this reason, the samples were sandwiched between two glass slides. Typically, the thickness of the sample was about $20 \mu \mathrm{m}$.

In order to simplify some kinetic studies, a femtosecond mode locked Ti/Sapphire laser with pulse duration around $100 \mathrm{fs}$ and $80 \mathrm{MHz}$ repetition rate was used as a tunable light source. The available wavelengths (690 to $1020 \mathrm{~nm}$ ) allowed a convenient screening of the wavelength dependence of the resin sensitivity and power of to $2 \mathrm{~W} / \mathrm{cm}^{2}$ at $800 \mathrm{~nm}$ could be achieved. The progress of the reaction was monitored via UV-visible absorption spectra using a Perkin-Elmer Lambda 2 spectrophotometer.

Transmission electron microscopy (TEM) was used to characterize the size and shape of Ag nanoparticles. The samples were irradiated directly onto the copper grid. TEM measurements were carried out using a Philips CM20 instrument with $\mathrm{LaB}_{6}$ cathode.

\section{Results and Discussion}

3.1. Fabrication of Silver Nanoparticles Embedded in a Polymer Matrix. A formulation of ethoxylated pentaerythritol tetraacrylate monomer from Sartomer containing the NIR sensitizer (0.1 wt $\%)$, MDEA ( $8 \% \mathrm{wt})$, and $\mathrm{AgNO}_{3}$ (1 wt $\%$ ) was photopolymerized upon irradiation at $800 \mathrm{~nm}$.

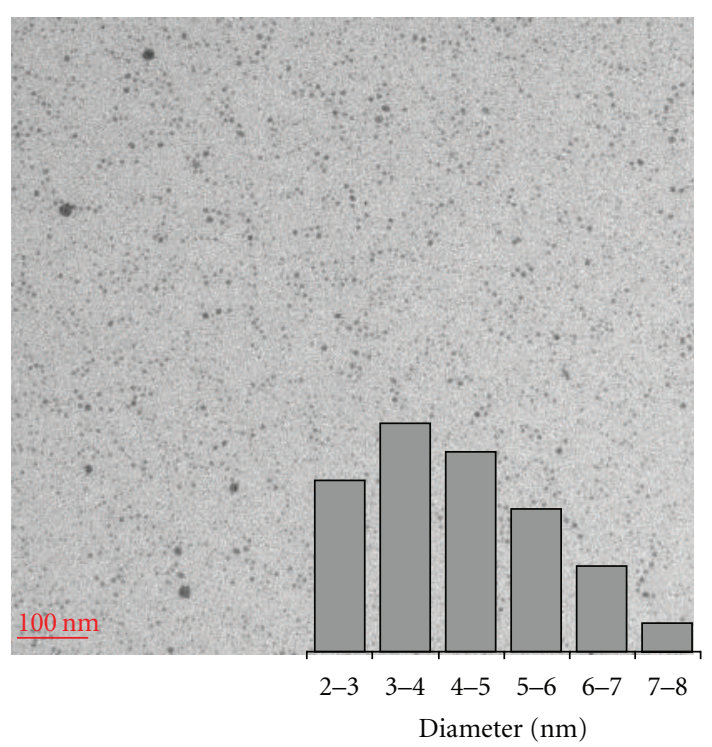

FIGURE 3: TEM image and the corresponding particle size distribution of silver nanoparticles in the polymer matrix obtained using S1 and intensity power equal at $300 \mathrm{~mW} / \mathrm{cm}^{2}$.

A followup of the stationary photolysis of the sensitizer/electron donor system was carried out with a view to confirming the initiation process of photopolymerization in the specific formulation used in this work. Figure 2(a) shows the time evolution of the absorption spectrum of a formulation irradiated with a light power of $300 \mathrm{~mW} / \mathrm{cm}^{2}$ at $800 \mathrm{~nm}$ with exposures ranging from 0 to $360 \mathrm{sec}$.

The bleaching of the sensitizer goes along with the development of a very weak absorption in the blue. This behavior is consistent with what is reported in the literature $[27,28]$. Upon NIR irradiation, the strong band corresponding to the absorption of the dye $(700-900 \mathrm{~nm})$ gradually faded. The major interest of this sensitizer is bleach upon irradiation (photobleaching), a feature which is of utmost importance for micro-optics fabrication, since the final material has to become transparent at the operating wavelength (typically ca $800 \mathrm{~nm})$.

When silver cations were present in the formulation (Figure 2(b)), the bleaching rate of the sensitizer was about twice as fast, and the well-known characteristic absorption band related to the plasmon of silver nanoparticles appeared in the blue region. This broad band with a quasigaussian shape has its maximum at $414 \mathrm{~nm}$ and a full-width-at-halfmaximum of ca $130 \mathrm{~nm}$.

TEM image (Figure 3) confirmed the fabrication of individual nanoparticles in the polymer matrix.

These nanoparticles are spherical with a diameter ranging from 2 to $7 \mathrm{~nm}$ as clearly visible on Figure 3. In spite of no addition of a capping agent to stabilize the nanoparticles, the TEM images show a good dispersion of the nanoparticles which are imprisoned and stabilized by the polymer network.

3.2. Effect of the Reaction Conditions on Silver Nanoparticles Embedded in the Polymer Matrix. The effect of irradiance 


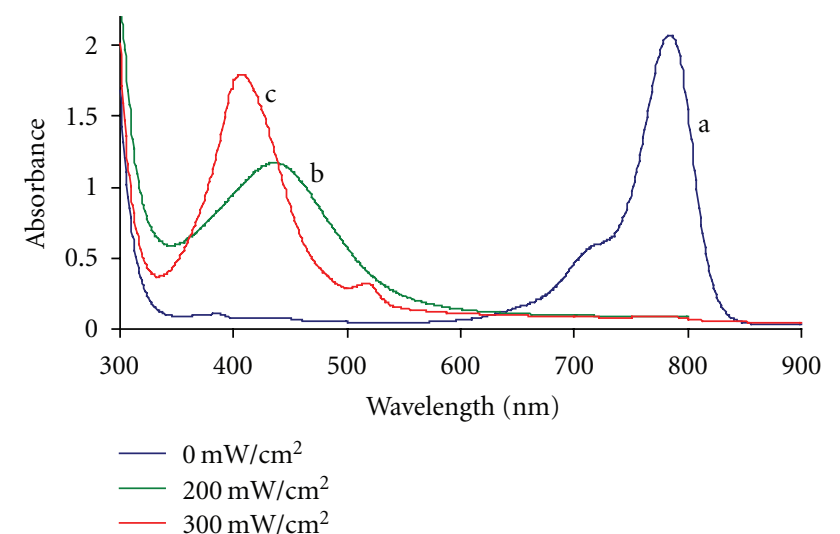

FIGURE 4: Time evolution of absorption spectra of the samples upon irradiation at $800 \mathrm{~nm}$ and using S1 (a) without silver cations, (b) with silver cations, incident light power $200 \mathrm{~mW} / \mathrm{cm}^{2}$, and (c) with silver cations, incident light power $300 \mathrm{~mW} / \mathrm{cm}^{2}$. Time irradiation $360 \mathrm{~s}$.

light power on nanoparticles growth was studied. Figure 4 shows the effect of power irradiation on the $\mathrm{Ag}^{+}$reduction by UV-Vis spectroscopy, in which the experiments were performed under the conditions of 0,200 and $300 \mathrm{~mW} / \mathrm{cm}^{2}$ in presence of $\mathrm{S} 1$.

No band appears in the region of $400-450 \mathrm{~nm}$ on the UV-Vis absorption spectrum of nonirradiated sample $\left(0 \mathrm{~mW} / \mathrm{cm}^{2}\right)$. At both $200 \mathrm{~mW} / \mathrm{cm}^{2}$ and $300 \mathrm{~mW} / \mathrm{cm}^{2}$, the brown yellow films were obtained accompanied with the appearance of absorption band at $440 \mathrm{~nm}$ and $414 \mathrm{~nm}$, respectively. Higher irradiance allows smaller and monodisperses particles in size to be generated. Irradiations at 200 and $300 \mathrm{~mW} / \mathrm{cm}^{2}$ produce nanoparticles of $14 \pm 1 \mathrm{~nm}$ and $4.5 \pm 0.9 \mathrm{~nm}$, as shown on Figures 5 and 3, respectively.

The sensitizer used to the reaction system could also affect the reduction rate of $\mathrm{Ag}^{+}$. UV-Vis spectroscopy (Figure 6) showed the effect of two different sensitizers used on the $\mathrm{Ag}^{+}$reduction while keeping the $\mathrm{AgNO}_{3}$ content unchanged. For the same reaction time and light power, $360 \mathrm{~s}$ and $300 \mathrm{~mW} / \mathrm{cm}^{2}$, the absorption peak intensity in the case of S2 it is very weak and broad compared with S1 (Figure 6).

\subsection{Effect of the Dye Sensitizer Structure on the Photoreduction} Reaction. Quite interestingly, both the kinetics of the MNPs generation and the size distribution of the particles can be related to structural effects of the dye sensitizer. Indeed, it is a well-established fact that any structural change likely to introduce some rigidization in the polymethine central chain of the sensitizer has the effect of decreasing singlet to singlet internal conversion due to mixing of the electronic and vibrational levels [29]. Consequently, the quantum yield of intersystem crossing to triplet increases in proportion that makes the reaction with electron donor (MDEA) and the photoreduction of silver precursors more efficient [30, 31]. Along the same lines, the heavy atom effect is also known to induce a significant increase of the triplet quantum yield.
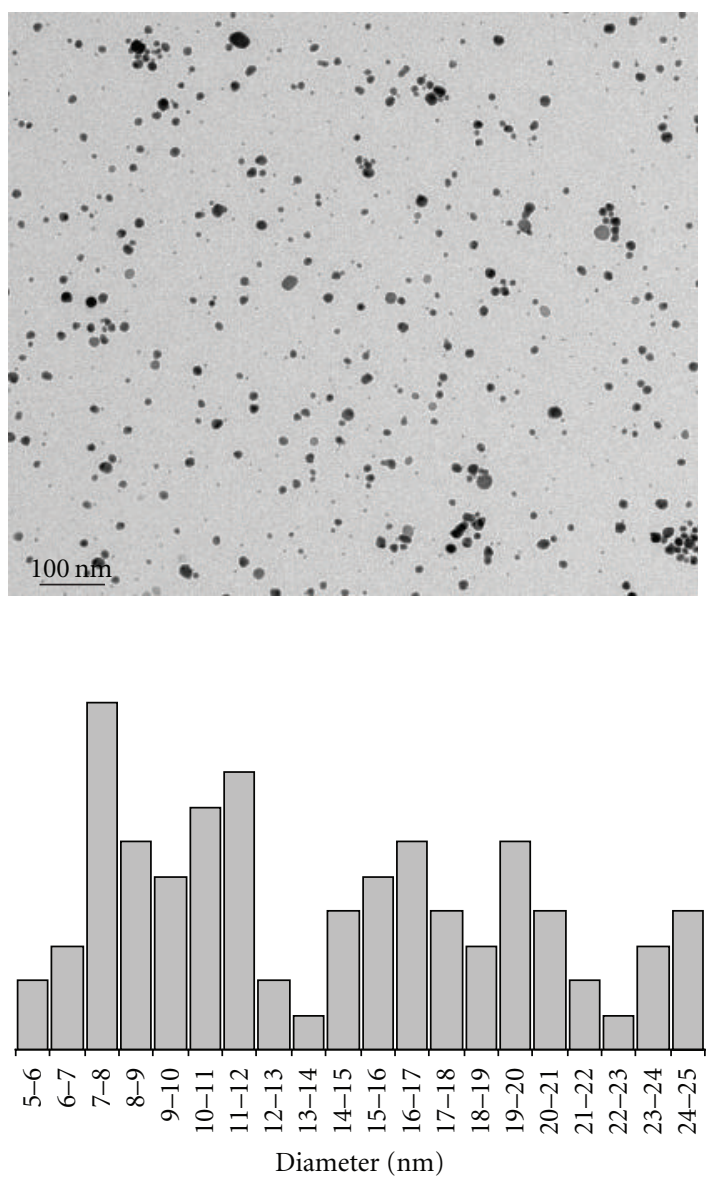

FIGURE 5: TEM image and the corresponding particle size distribution of silver nanoparticles in the polymer matrix obtained using S1 and intensity power equal at $200 \mathrm{~mW} / \mathrm{cm}^{2}$.

This effect is related to the presence of heavy atoms in the substituents carried by a given structure [32].

Thus, it is clearly visible from Figures 2 and 3 that the photoreduction kinetics is faster with S1 (the rigid polymethine structure and the structure carrying two chlorine atoms). Likewise, the absorption due to plasmon resonance of silver particles is weaker and much broader with S2 (the flexible polymethine structure) that denotes a large size distribution. These observations are consistent with a slower nucleation process that is propitious to size dispersion.

This analysis was corroborated by using other polymethine sensitizers, either with highly flexible central chain (2[7-(1,3-Dihydro-1,3,3-trimethyl-2H-indol-2-ylidene)hepta1,3,5-trienyl]-1,3,3-trimethyl-3H-indolium iodide), or with heavy atoms and a hydrocarbon bridge rigidifying the central chain (2-[2-[2-Chloro-3-[2-[1,3-dihydro-3,3-dimethyl-1(4-sulfobutyl)-2H-indol-2-ylidene]-ethylidene]-1-cyclohexen-1-yl]-ethenyl]-3,3-dimethyl-1-(4 sulfobutyl)-3H-indolium hydroxide, inner salt, sodium salt). The first one does not work at all, while the second one induces both photoreduction with a sharp size distribution of silver nanoparticles and crosslinking photopolymerization of the dispersion medium. 


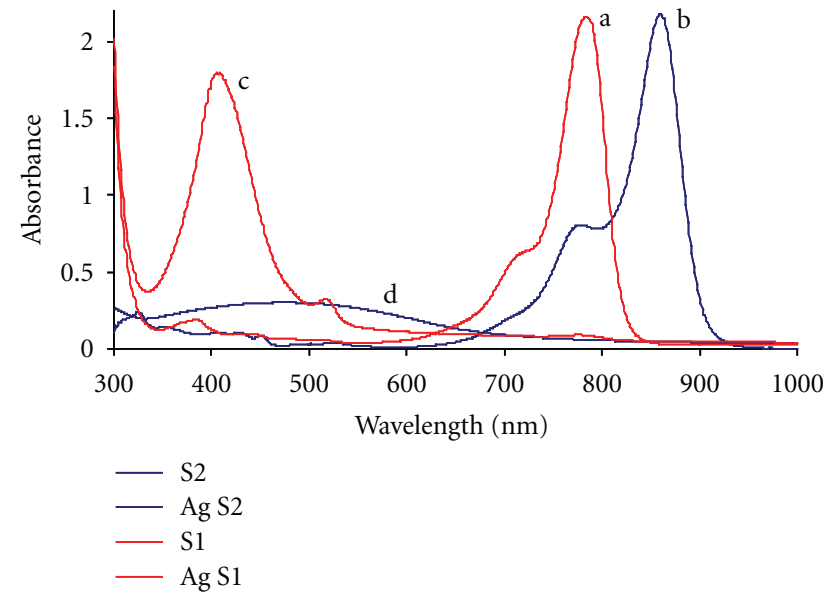

FIGURE 6: Time evolution of absorption spectra of the samples (a) with S1, exposure $0 \mathrm{~s}$, (b) with S2, exposure $0 \mathrm{~s}$, (c) with S1, exposure $360 \mathrm{~s}$ with an incident light power $300 \mathrm{~mW} / \mathrm{cm}^{2}$ upon irradiation at $800 \mathrm{~nm}$ and (d) with S2 exposure $360 \mathrm{~s}$ with an incident light power $300 \mathrm{~mW} / \mathrm{cm}^{2}$ upon irradiation at $850 \mathrm{~nm}$.

\section{Conclusions}

This work demonstrates the possibility of extending the concept of photochemically assisted synthesis of metal nanoparticles by direct reduction of metal ions or complex to the NIR range, a spectral region of crucial interest for in situ implementation of integrated optical functions but where the weak electronic energy carried by photons makes photochemical activation often critical.

A photopolymerizable formulation was tailored to allow initiation by the transient species used to reduce silver cations. It was found that a $300 \mathrm{~mW} / \mathrm{cm}^{2}$ light intensity forms metal nanoparticles homogeneously dispersed in corresponding polymer material, generating thus a metal/polymer nanocomposite that is fabricated upon NIR light activation. Specific effects of the structure of the polymethine sensitizers that strongly suggest the involvement of their triplet state were noticed.

This achievement should open up new vistas in several research fields, where the in situ photochemical synthesis of MNPs triggered by NIR might allow technical bottlenecks to be overcome.

\section{Acknowledgment}

The authors thank the Agence Nationale de la Recherche (ANR) for the financial support under Contract no. ANR09-JCJC-0029-01, Project PhotoPlasm.

\section{References}

[1] M. Sakamoto, M. Fujistuka, and T. Majima, "Light as a construction tool of metal nanoparticles: synthesis and mechanism," Journal of Photochemistry and Photobiology C, vol. 10, no. 1, pp. 33-56, 2009.

[2] R. G. Freeman, K. C. Grabar, K. J. Allison et al., "Selfassembled metal colloid monolayers: an approach to SERS substrates," Science, vol. 267, no. 5204, pp. 1629-1632, 1995.
[3] W. P. McConnell, J. P. Novak, L. C. Brousseau, R. R. Fuierer, R. C. Tenent, and D. L. Feldheim, "Electronic and optical properties of chemically modified metal nanoparticles and molecularly bridged nanoparticle arrays," Journal of Physical Chemistry B, vol. 104, no. 38, pp. 8925-8930, 2000.

[4] X. Duan, Y. Huang, Y. Cui, J. Wang, and C. M. Lieber, "Indium phosphide nanowires as building blocks for nanoscale electronic and optoelectronic devices," Nature, vol. 409, no. 6816, pp. 66-69, 2001.

[5] J. D. Holmes, K. P. Johnston, R. C. Doty, and B. A. Korgel, "Control of thickness and orientation of solution-grown silicon nanowires," Science, vol. 287, no. 5457, pp. 1471-1473, 2000.

[6] Y.-P. Sun, J. E. Riggs, H. W. Rollins, and R. Guduru, "Strong optical limiting of silver-containing nanocrystalline particles in stable suspensions," Journal of Physical Chemistry B, vol. 103, no. 1, pp. 77-82, 1999.

[7] Y. Dirix, C. Bastiaansen, W. Cased, and P. Smith, "Oriented pearl-necklace arrays of metallic nanoparticles in polymers: a new route toward polarization-dependent color filters," Advanced Materials, vol. 11, no. 3, pp. 223-227, 1999.

[8] H. Ditlbacher, J. R. Krenn, B. Lamprecht, A. Leitner, and F. R. Aussenegg, "Spectrally coded optical data storage by metal nanoparticles," Optics Letters, vol. 25, no. 8, pp. 563-565, 2000.

[9] J. Ouyang, C. W. Chu, D. Sieves, and Y. Yang, "Electric-fieldinduced charge transfer between gold nanoparticle and capping 2-naphthalenethiol and organic memory cells," Applied Physics Letters, vol. 86, no. 12, Article ID 123507, pp. 1-3, 2005.

[10] F. Hao, C. L. Nehl, J. H. Hafner, and P. Nordlander, "Plasmon resonances of a gold nanostar," Nano Letters, vol. 7, no. 3, pp. 729-732, 2007.

[11] D. A. Boyd, L. Greengard, M. Brongersma, M. Y. Ei-Naggar, and D. G. Goodwin, "Plasmon-assisted chemical vapor deposition," Nano Letters, vol. 6, no. 11, pp. 2592-2597, 2006.

[12] V. Sambhy, M. M. MacBride, B. R. Peterson, and A. Sen, "Silver bromide nanoparticle/polymer composites: dual action tunable antimicrobial materials," Journal of the American Chemical Society, vol. 128, no. 30, pp. 9798-9808, 2006.

[13] K. C. Anyaogu, A. V. Fedorov, and D. C. Neckers, "Synthesis, characterization, and antifouling potential of functionalized copper nanoparticles," Langmuir, vol. 24, no. 8, pp. 43404346, 2008.

[14] L. Balan and D. Burget, "Synthesis of metal/polymer nanocomposite by UV-radiation curing," European Polymer Journal, vol. 42, no. 12, pp. 3180-3189, 2006.

[15] L. Balan, R. Schneider, and D. J. Lougnot, "A new and convenient route to polyacrylate/silver nanocomposites by lightinduced cross-linking polymerization," Progress in Organic Coatings, vol. 62, no. 3, pp. 351-357, 2008.

[16] S. T. Selvan, J. P. Spatz, H. A. Klok, and M. Möller, "Gold-polypyrrole core-shell particles in diblock copolymer micelles," Advanced Materials, vol. 10, no. 2, pp. 132-134, 1998.

[17] M. K. Corbierre, N. S. Cameron, M. Sutton et al., "Polymerstabilized gold nanoparticles and their incorporation into polymer matrices [13]," Journal of the American Chemical Society, vol. 123, no. 42, pp. 10411-10412, 2001.

[18] P. K. Sudeep and P. V. Kamat, "Photosensitized growth of silver nanoparticles under visible light irradiation: a mechanistic investigation," Chemistry of Materials, vol. 17, no. 22, pp. 5404-5410, 2005.

[19] Y. Yagci, M. Sangermano, and G. Rizza, "In situ synthesis of gold-cross-linked poly(ethylene glycol) nanocomposites by 
photoinduced electron transfer and free radical polymerization processes," Chemical Communications, no. 24, pp. 27712773, 2008.

[20] L. Balan, M. Jin, J. P. Malval, H. Chaumeil, A. Defoin, and L. Vidal, "Fabrication of silver nanoparticle-embedded polymer promoted by combined photochemical properties of a 2,7diaminofluorene derivative dye," Macromolecules, vol. 41, no. 23, pp. 9359-9365, 2008.

[21] L. Balan, C. Turck, O. Soppera, L. Vidal, and D. J. Lougnot, "Holographic recording with polymer nanocomposites containing silver nanoparticles photogenerated in situ by the interference pattern," Chemistry of Materials, vol. 21, no. 24, pp. 5711-5718, 2009.

[22] L. Balan, J. P. Malval, R. Schneider, D. Le Nouen, and D. J. Lougnot, "In-situ fabrication of polyacrylate-silver nanocomposite through photoinduced tandem reactions involving eosin dye," Polymer, vol. 51, no. 6, pp. 1363-1369, 2010.

[23] N. Noiret, C. Meyer, and D. J. Lougnot, "Photopolymers for holographic recording. V. Self-processing systems with near infrared sensitivity," Pure and Applied Optics, vol. 3, no. 1, pp. 55-71, 1994.

[24] I. Bányász, D. J. Lougnot, and C. Turck, "Holography and holographic interferometry with a near IR sensitive photopolymer system," Journal de Physique III, vol. 7, no. 1, pp. 211222, 1997.

[25] T. Urano, H. Nagasaka, M. Shimizu, and T. Yamaoka, "Photopolymerization system thermally accelerated by a laser diode," Journal of Imaging Science and Technology, vol. 41, no. 4, pp. 407-412, 1997.

[26] A. Espanet, C. Ecoffet, and D. J. Lougnot, "PEW: photopolymerization by evanescent waves. II. Revealing dramatic inhibiting effects of oxygen at submicrometer scale," Journal of Polymer Science Part A, vol. 37, no. 13, pp. 2075-2085, 1999.

[27] D. J. Lougnot, S. Lepaja, J. P. Fouassier, and J. Faure, "Etude physicochimique d'une série de cyanines. Partie V: le mécanisme de photodégradation," Journal de Chimie Physique et de Physicochimie Biologique, vol. 79, no. 9, p. 618, 1982.

[28] S. Lepaja, H. Strub, and D. J. Lougnot, "Photophysical study of a series of cyanines. Part III: The direct photooxidation reaction," Zeitschrift für Naturforschung A, vol. 38a, no. 1, pp. 56-60, 1983.

[29] P. Czerney, G. Graneß, E. Birckner, F. Vollmer, and W. Rettig, "Molecular engineering of cyanine-type fluorescent and laser dyes," Journal of Photochemistry and Photobiology A, vol. 89, no. 1, pp. 31-36, 1995.

[30] J. P. Fouassier, D. J. Lougnot, and J. Faure, "Photoisomerization processes in the IR-140 laser dye," Optics Communications, vol. 23, no. 3, pp. 393-397, 1977.

[31] C. Carré, C. Reichardt, and D. J. Lougnot, "Etude physicochimique d'une série de cyanines: Partie VII: Rendement en état triplet et photosensibilisation de l'oxygène singulet," Journal de Chimie Physique et de Physicochimie Biologique, vol. 84, no. 4, pp. 577-585, 1984.

[32] K. Jia, Y. Wan, A. Xia, S. Li, F. Gong, and G. Yang, "Characterization of photoinduced isomerization and intersystem crossing of the cyanine dye Cy3," Journal of Physical Chemistry A, vol. 111, no. 9, pp. 1593-1597, 2007. 

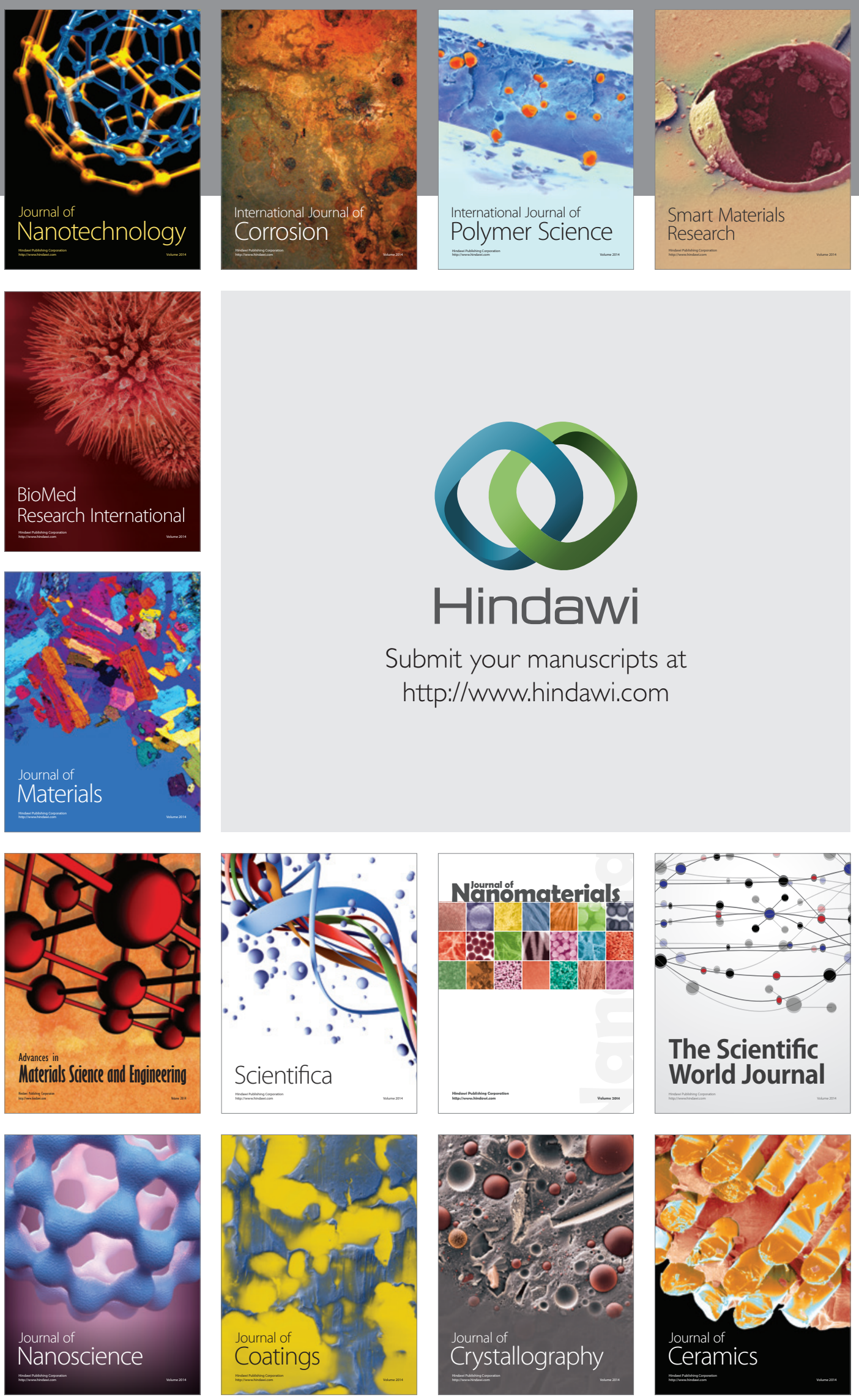

The Scientific World Journal

Submit your manuscripts at

http://www.hindawi.com

\section{World Journal}

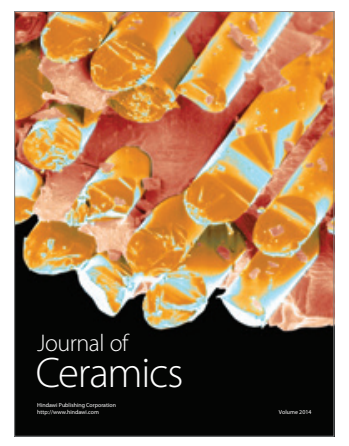

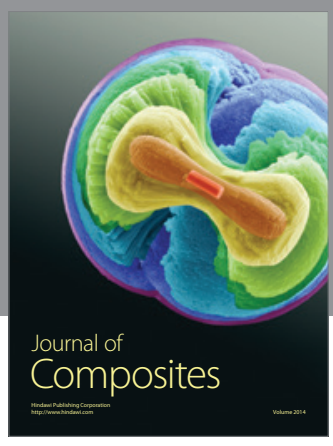
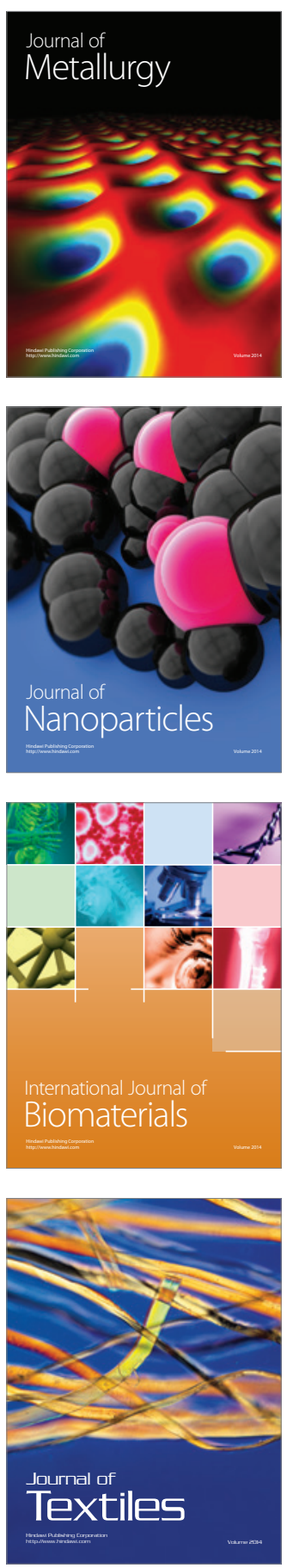